\title{
Combining Fast Response and Low Cost in an Intensive Care Unit Viewing Station
}

\author{
Erwin Bellon, Wilfried Houtput, Bart Bijnens, Paul Suetens, Guy Marchal, and Peter Lauwers
}

\begin{abstract}
This paper describes a prototype viewing station for the Intensive Care Unit (ICU) that combines a low cost with a fast response for the primary viewing tasks. A single monitor is used together with the possibility to switch to another image on the same screen instantaneously. To reduce the time for image retrieval and display, the viewing station anticipates the need for particular images, and preloads these from the disk into the internal memory whenever the computer has some idle time. Preliminary experience suggests that the use of a single monitor is no fundamental limitation for this application. Most of the time, the system can anticipate which images will be required shortly. This has an important impact on the user efficiency. Copyright 1994 by W.B. Saunders Company
\end{abstract}

KEY WORDS: Intensive care unit, computer systems, viewing stations, picture archiving and communication systems, image management.

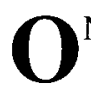

NE OF THE APPLICATIONS where a picture archiving and communication system (PACS) has the potential to improve working efficiency and patient care is that of image handling and viewing in intensive care units (ICUs). The growing use of computed Radiography for the ICU application is a beneficial factor for the introduction of PACS, by facilitating the access to high-quality digital images. One advantage of the electronic distribution of ICU images is that diagnostic information is likely to be more quickly available at the ward, although this may require changes in the relation between the radiologist who performs the primary diagnosis and the ICU clinician. ${ }^{1-5}$

Because in our particular situation, the traditional film-based working method has been highly optimized to provide a prompt service to the ICU, we do not expect the aspect of speed to be the most important advantage of PACS to our institution. Other possible improvements include a higher efficiency in handling the images accumulated during the stay of the patient, and the simultaneous availability of previous images for primary diagnosis in the department of radiology as well as for consultation at the ward.

Currently, a fundamental limiting factor in the introduction of PACS is the associated cost, especially when there is no clear-cut advantage with respect to patient care. A large part of the equipment cost can be attributed to the viewing stations. ${ }^{6}$ The commercially available lowerbudget systems often lack the required speed or 'responsiveness', as it takes several seconds to display an image on the screen. When a digital system must compete with a well-organized film based system, this aspect of user efficiency is critical. On the other hand, state-of-the-art digital equipment can supply the required speed, but at a very high cost. The technological requirements and thus the cost may be lower for secondary reviewing stations at the ward than for the viewing stations for primary diagnosis. Nevertheless, for a successful introduction of PACS, both types of viewing stations should have a high efficiency-cost ratio.

In this paper, we focus on concepts that allow us to obtain a workable combination of low cost and high responsiveness using widely available commercial components that are not on the cutting edge of technology. In a first stage, we developed a chest image viewing station for the ICU ward, but the concepts of this viewing station can be translated into a workstation for primary diagnosis as well.

The ICU viewing station is equipped with a single monitor, using fast 'swapping' when two images must be compared. To reduce the time for image retrieval and display, we have implemented an image preloading scheme. Essentially, the viewing station takes advantage of the short time periods during which the interactive user is not requesting action by the computer (eg, when studying the image currently on screen) to preload from the slower disk into the

From the Departments of Electrical Engineering and Radiology, Interdisciplinary Research Unit for Radiological Imaging; the Departments of Cardiology and Intensive Care Medicine, University Hospital Gasthuisberg; and Katholieke Universiteit Leuven, Belgium.

Address reprint requests to Enwin Bellon, ESAT and Radiology, Herestraat 49, 3000 Leuven, Belgium.

Copyright $\odot 1994$ by W.B. Saunders Company 0897-1890/94/0702-0019\$3.00/0 
fast internal memory, those images that probably will have to be displayed shortly. When one of these images is actually needed, it can be shown immediately. To let the system anticipate which images should be preloaded, a distinction was made between the different working modes that are most frequently used by the ICU physicians.

In this paper, we first motivate the approach that has been taken for image preloading. Then, we describe the demonstrator system that has been developed for the experiments. Finally, we discuss the results of initial tests.

\section{IMAGE PRELOADING TO SUPPORT DIFFERENT REVIEW MODES}

The image preloading scheme takes advantage of the fact that the images are often viewed in a specific order. This order depends on the particular viewing task. We have considered user efficiency to be the most important in the following viewing modes: the structured review mode, where the newly acquired images of all patients in the ward are studied consecutively and compared with the previous images; the emergency review mode, where a clinician drops in for a moment to quickly check a detail on the most recent image of a particular patient; and the less frequently used historic review mode, where the images of one particular patient accumulated over a longer period are studied.

\section{Structured Review Mode}

In structured review mode, it is customary to study the two most recent images of all patients in the ward. The viewing station uses this information to keep image display times low as well as to optimize the user interface. It still takes some time for the system to load and display the very first image. However, while the clinician is viewing this first image, the system loads the next images into the internal memory, as far as the available memory and the idle time allow (Fig 1). Once the last two images of a patient are available in memory, the user can rapidly swap between them by pressing a mouse button. When he switches to the next patient, the images of the previous patient are flushed to make memory available to preload further images.
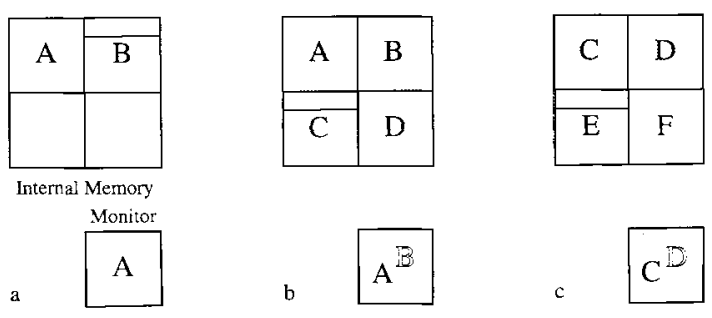

Fig 1. In structured review mode, the system tries to preload a number of images in internal memory (eg, two per patient). While the first image of the first patient is being displayed, a second image is prepared in the background (a). Two images that are in memory can be exchanged on the monitor instantaneously (b). When moving to the next patient, the images are immediately available (c). Memory taken by previous images is reused to preload further images.

\section{Emergency Review Mode}

For the emergency review mode, the user will not engage in a relatively long and coordinated session with the viewing station. Although the delay before the first image is displayed is not a major problem in structured review mode, it cannot be tolerated in the emergency review mode. Therefore, whenever the viewing station is not being used for any viewing, it automatically prepares a possible request for emergency review by preloading the most recent images of all patients in the ward. The memory required to hold one image of ten or fifteen patients is relatively inexpensive, when compared to the cost of high-speed magnetic disks or similar external storage devices. These images are presented on the screen as picture icons, sorted according to the bed number (Fig 2). To view any of these images, it is sufficient to click the corresponding icon image in the overview. This reduces the number of manual actions to the minimum.

\section{Historic Review Mode}

In the historic review mode, predicting which images will be viewed next is done in a way similar to the structured review mode.

\section{PILOT SYSTEM SET-UP}

The demonstrator system that has been constructed for a first evaluation of these concepts consists of an IMLOGIX 2000 display unit (Microterm Inc, St Louis, MO) controlled by a common personal computer (PC) (Fig 3).

The IMLOGIX display unit consists of the actual screen and internal video memory. The 


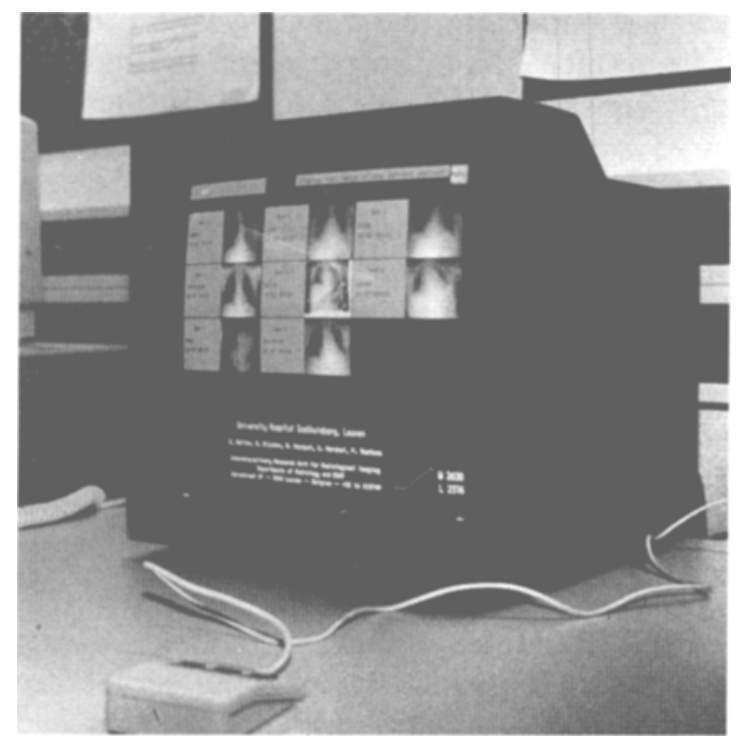

Fig 2. For emergency review mode, the user can directly request any of the most recent images by clicking the corresponding picture icon. If the system has been left idle for several tens of seconds, it can present the requested image immediately.

screen has a resolution of $1,024 \times 1,024$ pixels, but the internal memory is large enough to hold a number of such images (four in the configuration that was used). This display unit supports gray-value windowing using a 12-bit video lookup table, of which only 8 bits were used. The PC controls which 1,024- $\times$ 1,024-pixel part of the video memory is actually displayed on the screen;

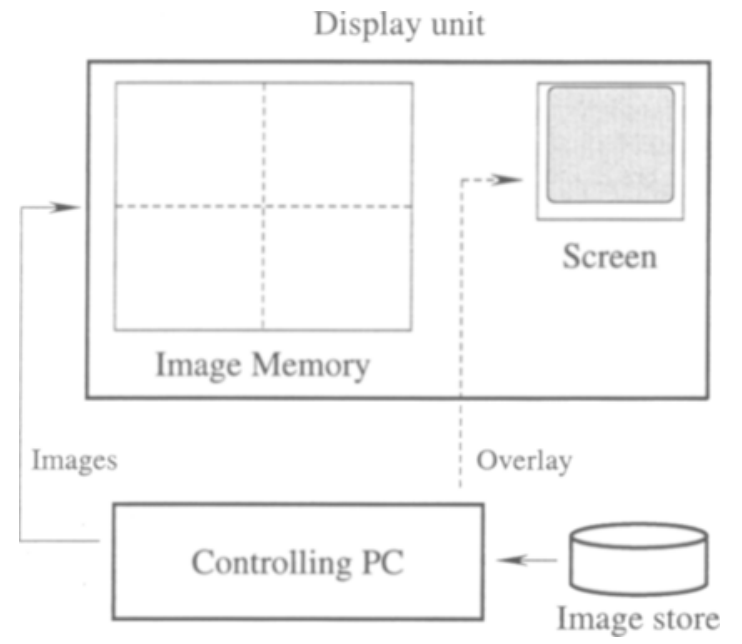

Fig 3. Schematic set-up of the ICU viewing station. The figure shows the relation between the display screen, the fast display memory (of which a part can be visible on the screen). and the controlling PC. it presents menus on the screen and loads images from the magnetic disk into the video memory. These images are transferred from the disk to the video memory in small parts, so that response to user interaction is not slowed down by the background process of image loading. Menus and control buttons are presented on the same monitor as the image display. All user interaction can be performed using the mouse only, without the need for keyboard input.

With the prototype set-up that was used, it is not possible to store enough images simultaneously in the internal memory to directly support the emergency review mode. However, we get around this by loading lower-resolution (512- $\times$ 512-pixel) versions of the images in the memory and by taking advantage of the realtime zooming capabilities of this particular display unit. This lower-resolution image can be presented immediately, and it is automatically replaced after a few seconds by the image at full resolution. Replacing the lower-resolution image (zoomed twice) by the higher-resolution image is almost unnoticeable.

In the set-up described here, the $\mathrm{PC}$ requires little memory. However, it would have been possible to use an unintelligent monitor, to put the image memory in the $\mathrm{PC}$, and to have the $\mathrm{PC}$ handle the windowing task.

\section{EXPERIMENTAL EVALUATION AND DISCUSSION}

The viewing station has been tested in one 12-bed ward. Chest images were acquired using a prototype phosphor plate scanner (ADC, Agfa-Gevaert NV, Mortsel, Belgium) and reduced to 1,024- $\times 1,024$-pixel resolution. For 2 months, the chest radiologist responsible for the ICU used the system in the structured review mode when explaining his findings at ICU. During this period, hardcopy films remained available. Later on, the viewing station was used for 3 weeks as the primary system, whereas the hardcopy films were only available in sealed envelopes.

Although a very slow disk has been used in this experiment, needing approximately $20 \mathrm{sec}$ onds to load an image, the users considered the waiting times for image display totally acceptable. The image preloading scheme is clearly efficient for the structured and the historical 
review modes, where waiting times were almost reduced to zero in most situations. For the emergency review mode, the speed of image access was considered sufficient, with no objection with respect to initial low-resolution (512- $\times$ 512-pixel) presentation. However, the experiment may have been too short to be conclusive about all aspects of the emergency review.

The success of the image-preloading scheme illustrates that, in the current stage of PACS technology, it pays off to try and compensate for the relatively unfavorable performance of standard components by using software workarounds specially adapted to the particular clinical organization.

The clinicians have not considered it an important drawback that only one monitor is available, as long as it is possible to switch rapidly to another image on the same screen. However, we have not performed tests to measure whether sequential display is as efficient as simultaneous display (using two screens). Some clinicans would probably have preferred a twoscreen system, if they had been given the choice. These informal findings with respect to using a single monitor are in agreement with preliminary results. ${ }^{7,8}$ In our experiment, the radiologists considered a single screen with the fastswap option sufficient. This is particularly encouraging in view of primary diagnosis on screen, because for this task, a resolution higher than $1,024 \times 1,024$ pixels may be indicated. Then, the monitor is an important factor in the cost of the viewing station.

The system that has been described in this report was only intended to check the validity of the basic concepts. A particular problem with using simple PCs is the software development cost required to come to a comprehensive system. Therefore, we have started the development of a more production-oriented viewing system based on a low-budget UNIX computer with industry-standard display hardware and software. This system will be closely linked to the radiology information system for automating most of the image management tasks.

\section{ACKNOWLEDGMENT}

The authors acknowledge the support of the companies Agfa-Gevaert NV, Mortsel, Belgium, and Microterm GmbH, Mönchengladbach, Germany.

\section{REFERENCES}

1. Humphrey LM, Fitzpatrick K, Atallah N, et al: Time comparison of intensive care units with and without digital viewing systems. J Digit Imaging 6:37-41, 1993

2. Kundel HL, Seshadri SB, Arenson RL: Clinical evaluation of PACS at the University of Pennsylvaria, in Huang HK, Ratib O, Bakker AR, Witte G (eds): Picture Archiving and Communication Systems (PACS) in Medicine. NATO ASI Series F (Computer and Systems Sciences), vol 74. New York, NY, Springer-Verlag, 1991, pp 195-200

3. Gillespy T, Staab EV, Lawrence E: Electronic imaging in a teaching hospital intensive care unit: Evaluation of the clinical review system. J Digit Imaging 3:124-128, 1990

4. De Simone DN, Kundel HL, Arenson RL, et al: Effect of a digital imaging network on physician behaviour in an intensive care unit. Radiology 169:41-44, 1988
5. Mezrich RS: The implication of PACS for radiology practice. Am J Roentgenol 151:828, 1988

6. Saarinen AO, Haynor DR, Loop JW, et al: Modeling the economics of PACS: What is important? Proceedings of the SPIE (Medical Imaging III: PACS System Design and Evaluation) 1093:62-73, 1989

7. Carboni NL, Tombaugh JW, Dillon RF: The effect of simultaneous vs sequential viewing of digital images on comparative judgment performance. Proceedings of the SPIE (Medical Imaging III: Image Capture and Display) 1091:252-261, 1989

8. Gurr D, Good WF, King JL: Simultaneous and sequential display of ICU AP-chest images. Proceedings of the SPIE (Image Capture, Formatting, and Display) 1653:159163,1992 